\title{
THE INVESTIGATION INTO PRIMACY EFFECT ON STUDENT'S VOCABULARY MEMORIZATION
}

\author{
Delli Sabudu \\ Manado State University \\ dellisabudu@unima.ac.id
}

\begin{abstract}
This study aimed to examine whether primacy positions are more dominant in memorizing words, in other words, this study intends to test which ones are easier to remember words that are at the top or the beginning or words that are at the bottom or end in the word memorization list. In this study, the authors used a sample of first grade SMP Negeri I students. From a number of parallel classes the author takes the class as a sample. Data collection is done through tests in the form of a post-test. By using the t-test formula, the collected data is then analyzed to test the hypothesis whether there is a significant difference between the two groups of students who memorize the primacy position and the students who memorize the recency position. The experimental group gets a higher number in memorizing words in the primacy position in memorizing vocabulary. By using a two-tailed test, proved that Ho is rejected. For this reason, it is expected that teachers who teach English or other foreign languages can teach vocabulary by placing difficult words on the memorizing list in the top or primary position.
\end{abstract}

Keywords: Primacy, Recency, vocabulary, Memorization.

\section{INTRODUCTION}

There are various reasons why we remember words better than others. The nature of words affects our memory (Ur, 1996). Words consisting of one or two syllables are easier to remember that those of three or more. Words referring to concrete objects are easier to remember that those referring to abstract nouns. Another reason why we remember words is circumstances we are learnt. Words learnt in relax are easier to remember than those learnt under pressure. Method of teaching also is another reason. Words taught visually are easier to remember than words taught through verbal illustrations.

Whether words are easier or difficult to remember is determined by their position in a word list. Research findings show that when given a word list and we are memorized the words in the period of time, those come at the top of the list are easier to remember than those occupying mid or final position. The words in top position are the first that catch out attention, than those in the mid and final position. Effect of top position is called "primacy effect" (Good \& Broophy, 1982:218).

Research evidences also show that words come at the end position of word list are easier to remember than those in the top and mid position. They come with the reason that when a given word list is memorized, the word in the final position is the latest

CELTIC: A Journal of Culture, English a Teaching, Literature \& Linguistics ISSN: 2356-0401, E-ISSN: 2621-9158, VOL. 6, NO. 1, June 2019. 
information that enters our memory before. Words in the final position interfere in our memory (Walker 1996). Still another research evidences indicate that words in both top and final position are easier to remember than those in the mid position. The reason is, as Mayer (1998:2) points out, “...because items at the beginnings and ends of lists serve as cognitive landmarks that provide anchors to which the other items may be attached in memory".

Based on the three ideas about the above effects on students' memorization in vocabulary, the writer would like to find out which of those ideas or theory is dominant in vocabulary memorization and that is why this study is done to investigate the cause. The purpose of this study is particularly intended to find out whether the recency effect is more dominant in word recall than primacy effect? While the hypothesis itself that primacy effect is more dominant than recency effect in word recall. The students that choose as a sample is assumed to have normal memory and the data in the form of test scores are normally distributed.

The information that was provided by this study is particularly important for a number of people: theoreticians in information processing, researchers in foreign language teaching and teachers of English as a foreign language.

\section{Memorizing Vocabulary}

Vocabulary can be defined, roughly, as the words we teach in the foreign language (Ur, 1996). Vocabulary can be acquired in many different ways. One way is through word memorization. The words in your vocabulary are those you understand and use."

Word memorization actually in the form of memory experiment, it involves the recall of as many items as possible on a word list. The words, which are put in the word-list may be taken from a dictionary or reading text in the textbooks. Students are given lists of target language vocabulary words and their native language equivalents, then students are required to memorize them and tested regularly. Generally, the memorized words can be easily kept in our memory, but sometimes they are easily forgotten.

There are a number of reasons why words memorized from word list are easy or difficult to be held in our memory. Words are easily kept or held in our memory and can be easily retrieved because we have already had similar knowledge or experience related to the words we memorize. They can be easily forgotten because we do not have previous knowledge related to the words.

Position of words in a list play is the most important part in determining whether or not given word is easy or difficult to be held in our memory and recalled. Murdock, as quoted by Walker (1996), found that the first and the last portions in word list are learned more readily than the middle. He also found that the last portion is easier to be kept in our memory and recalled than the first portion. Ruch (1984) also points out the

CELTIC: A Journal of Culture, English a Teaching, Literature \& Linguistics

ISSN: 2356-0401, E-ISSN: 2621-9158, VOL. 6, NO. 1, June 2019. 
same thing. He states that if a list consisting of 20 words is to be memorized, the first and the last portions are better recalled than the middle portion.

Why can the first and the last portions be the first and the last words that we first and last see? When we are given a word list, we tend to pay minimal attention to those mentioned in the middle of the list. That is the reason why the first and the last portions of words in the word lists are easier to be memorized and recalled. The effects of the first and the last portion are called primacy and recency effects.

\subsection{Primacy Effect}

Primacy Effect can be explained as the effect that happens while the items are arranged in the word lists; for example, list the names and try to memorize them. After you write down the names again, you can see that the names in the top position can be memorized better. This happens because the top position is prior learning (pro-active inhibition) (Holzman, 1978).

\subsection{Recency Effect}

Recency effect can be explained as the effect that happens while the items arranged in a list. After students memorize the items, then you can find that the items in the last position can be better memorized. This happens because what we learn recently may interfere with what we have learned before. In this case, the words or items in the last portion interfere the recall of words learned before. This is what Walker (1996: 222) calls "retroactive inhibition".

\section{Statistical Formulation}

In this study, the writer applied the t-test formula in order to compare the ability of the two groups. Basically, this statistical formula follows the assumption of homogeneity of variance, where:

(1)The scores in the two groups are randomly sampled how their respective population and are independent of one another.

(2)The scores in the respective, populations are normally distributed.

(3)The variances of scores in the population are equal to the independent variable positively affects the dependent variable. To prove whether the hypothesis was rejected or not, the t-test formula by Shavelson (1981) was used.

$$
\mathrm{t}_{\mathrm{x}-\mathrm{x}}(\mathrm{obs})=\frac{\overline{\mathrm{X}}_{1}-\overline{\mathrm{X}}_{2}}{\sqrt{\left[\frac{\left(\mathrm{n}_{1}-1\right) \mathrm{S}_{1}^{2}+\left(\mathrm{n}_{2}-1\right) \mathrm{S}_{2}^{2}}{\mathrm{n}_{1}+\mathrm{n}_{2}-2}\right]\left[\frac{1}{\mathrm{n}_{1}}+\frac{1}{\mathrm{n}_{2}}\right]}}
$$

\section{Where :}

$\mathrm{x}_{1} \quad=$ Mean score of experimental group

$\mathrm{x}_{1}=$ Mean score of control group

$\mathrm{n}_{1}=$ Total number of the experimental group

$\mathrm{n}_{2}=$ Total number of the control group 
$\mathrm{S}_{1}^{2}=$ Calculation of variance of the experimental group

$\mathrm{S}_{2}{ }^{2}=$ Calculation of variance of the control group to test the

hypothesis, the following formula ratio is used :

$$
\begin{array}{cc}
\mathrm{H}_{\mathrm{o}}= & \mu_{1}=\mu_{2} \\
\mathrm{H}_{1}= & \mu_{1} \neq \mu_{2} \\
\mathrm{~A}= & .05
\end{array}
$$

The criteria for rejecting the null hypothesis are:
(a) Do not reject $\mathrm{H}_{\mathrm{o}}$
if $t_{o b s}<t_{\text {erit }}$
(b) Reject $\mathrm{H}_{\mathrm{o}}$
if $\mathrm{t}_{\mathrm{obs}}<\mathrm{t}_{\text {erit }}$

(This means that the alternative hypothesis is accepted)

\section{FINDINGS AND DISCUSSION}

To prove whether the hypothesis is rejected or not, the writer conducted the TrueExperimental Research. The data were concerned with the use of memory-test. It was in the form of a plain answer sheet, in which the subjects were to write down all the words they had memorized previously from a word list. The memory test was a post-test which measures the effect of the independent variable on the dependent variable.

The population of this research was the students in the first grade of SLTP Negeri I Manado, which consisted of 10 classes. One of the ten classes was chosen randomly and decided to be the sample class. This sample class consisted of 48 students and 40 were randomly chosen as subjects of this research.

18 words in the list were divided into three positions that is six on the top position, which is called primacy position, six in the second position which is called the middle, and six in the last position, which is called recency position. The primacy was the Experimental group and Recency as the Control group. The treatment was the position of words in the word list.

Based on the hypothesis, there were two groups in the research. To find out the difference between the two groups, the t-test formula was used to test.

In order to analyze the data, the writer used the following steps :

Step 1: Checking the result of post-test in the Experimental Group and the control group.

Step 2: $\quad$ Checking the frequency distribution of the Experimental group and Control group.

Step 3: Calculating the result of the post-test in the Experimental and Control group.

Step 4: Computing $\mathrm{t}_{\mathrm{x} 1-\mathrm{x} 2}$ by inserting the appropriate values into the $\mathrm{t}$-test formula.

Step 5: Deciding whether to reject the null hypothesis or not.

The following is the result of post-test in the Experimental group and Control group. 
Table1. Result of the post-test in the Experimental group and Control group

\begin{tabular}{|c|c|c|c|}
\hline $\mathrm{n}_{1}$ & $\mathrm{x}_{1}$ & $\mathrm{n}_{2}$ & $\mathrm{x}_{2}$ \\
\hline$\overline{c 1}$ & $\bar{~} 6$ & $\overline{c 1}$ & $\overline{55}$ \\
\hline 2 & 5 & 2 & 4 \\
\hline 3 & 6 & 3 & 5 \\
\hline 4 & 4 & 4 & 3 \\
\hline 5 & 6 & 5 & 5 \\
\hline 6 & 5 & 6 & 2 \\
\hline 7 & 5 & 7 & 4 \\
\hline 8 & 6 & 8 & 5 \\
\hline 9 & 5 & 9 & 4 \\
\hline 10 & 6 & 10 & 5 \\
\hline 11 & 3 & 11 & 0 \\
\hline 12 & 5 & 12 & 4 \\
\hline 13 & 6 & 13 & 5 \\
\hline 14 & 6 & 14 & 4 \\
\hline 15 & 6 & 15 & 5 \\
\hline 16 & 5 & 16 & 4 \\
\hline 17 & 5 & 17 & 4 \\
\hline 18 & 5 & 18 & 4 \\
\hline 19 & 5 & 19 & 2 \\
\hline 20 & 6 & 20 & 3 \\
\hline 21 & 5 & 21 & 2 \\
\hline 22 & 6 & 22 & 3 \\
\hline 23 & 4 & 23 & 3 \\
\hline 24 & 6 & 24 & 4 \\
\hline 25 & 6 & 25 & 5 \\
\hline 26 & 5 & 26 & 4 \\
\hline 27 & 6 & 27 & 3 \\
\hline 28 & 5 & 28 & 3 \\
\hline 29 & 5 & 29 & 5 \\
\hline 30 & 5 & 30 & 5 \\
\hline
\end{tabular}

Table 1 continued.

\begin{tabular}{|c|c|c|c|}
\hline $\mathrm{n}_{1}$ & $\mathrm{x}_{1}$ & $\mathrm{n}_{2}$ & $\mathrm{x}_{2}$ \\
\hline 31 & 3 & 31 & 6 \\
32 & 6 & 32 & 6 \\
33 & 6 & 33 & 6 \\
34 & 6 & 34 & 6 \\
35 & 6 & 35 & 2 \\
36 & 2 & 36 & 6 \\
37 & 4 & 37 & 5 \\
38 & 3 & 38 & 6 \\
39 & 5 & 39 & 5 \\
40 & 4 & 40 & 5 \\
\hline
\end{tabular}

CELTIC: A Journal of Culture, English a Teaching, Literature \& Linguistics

ISSN: 2356-0401, E-ISSN: 2621-9158, VOL. 6, NO. 1, June 2019. 


\begin{tabular}{l|l|l|l|}
$\Sigma \mathrm{n}_{1}=40$ & $\Sigma \mathrm{n}_{1}=204$ & $\Sigma \mathrm{n}_{2}=40$ & $\Sigma \mathrm{n}_{2}=165$ \\
\hline
\end{tabular}

Table 2. Frequency Distribution of the Experimental Group $\left(\mathrm{x}_{1}\right)$

\begin{tabular}{|c|c|c|}
\hline $\begin{array}{c}\text { Score Value } \\
\left(\mathrm{n}_{1}\right)\end{array}$ & Tally & $f$ \\
6 & & 17 \\
5 & & 15 \\
4 & & 4 \\
3 & & 3 \\
2 & & 1 \\
\hline
\end{tabular}

Table 3. Frequency Distribution of the Control Group $\left(\mathrm{x}_{2}\right)$

\begin{tabular}{|c|c|c|}
\hline $\begin{array}{c}\text { Score } \\
\text { Value } \\
\left(\mathrm{n}_{2}\right)\end{array}$ & Tally & $f$ \\
6 & & 6 \\
5 & & 12 \\
4 & & 10 \\
3 & & 7 \\
2 & & 4 \\
1 & & 1 \\
\hline
\end{tabular}

Table 4. Calculation of the Result of the Post-test in Experimental Group $\left(\mathrm{x}_{1}\right)$ 


\begin{tabular}{|c|c|c|c|c|}
\hline $\mathrm{n}$ & $\mathrm{x}_{1}$ & $\overline{\mathrm{X}}_{2}$ & $\mathrm{x}_{1}-\overline{\mathrm{x}}_{1}$ & $\left(\mathrm{x}_{1}-\overline{\mathrm{x}}_{2}\right)^{2}$ \\
\hline 11 & 6 & $\begin{array}{l}5.1 \\
\end{array}$ & 9.9 & .81 \\
\hline 2 & 5 & 5.1 & -.1 & -.2 \\
\hline 3 & 6 & 5.1 & .9 & .81 \\
\hline 4 & 4 & 5.1 & - & -2.2 \\
\hline 5 & 6 & 5.1 & .1 & .81 \\
\hline 6 & 5 & 5.1 & .9 & .01 \\
\hline 7 & 5 & 5.1 & -.1 & .02 \\
\hline 8 & 6 & 5.1 & -.1 & .81 \\
\hline 9 & 5 & 5.1 & .9 & .02 \\
\hline 10 & 6 & 5.1 & -.1 & .81 \\
\hline 11 & 3 & 5.1 & -.9 & -4.2 \\
\hline 12 & 5 & 5.1 & - & -.2 \\
\hline 13 & 6 & 5.1 & .1 & .81 \\
\hline 14 & 6 & 5.1 & -.1 & .81 \\
\hline 15 & 6 & 5.1 & .9 & .81 \\
\hline 16 & 5 & 5.1 & .9 & .2 \\
\hline 17 & 5 & 5.1 & .9 & .2 \\
\hline 18 & 5 & 5.1 & -.1 & .2 \\
\hline 19 & 5 & 5.1 & -.1 & .2 \\
\hline 20 & 6 & 5.1 & -.1 & .81 \\
\hline 21 & 5 & 5.1 & -.1 & -.2 \\
\hline 22 & 6 & 5.1 & .9 & .81 \\
\hline 23 & 4 & 5.1 & -.1 & -2.2 \\
\hline 24 & 6 & 5.1 & .9 & .81 \\
\hline 25 & 6 & 5.1 & 1.1 & .81 \\
\hline 26 & 5 & 5.1 & .9 & -.2 \\
\hline 27 & 6 & 5.1 & .9 & .81 \\
\hline 28 & 5 & 5.1 & -.1 & .2 \\
\hline 29 & 5 & 5.1 & .9 & -.2 \\
\hline 30 & 5 & 5.1 & -.1 & -.2 \\
\hline 31 & 3 & 5.1 & -.1 & -4.2 \\
\hline 32 & 6 & 5.1 & -.1 & .81 \\
\hline 33 & 6 & 5.1 & .9 & .81 \\
\hline 34 & 6 & 5.1 & .9 & .81 \\
\hline 35 & 6 & 5.1 & .9 & .81 \\
\hline 36 & 2 & 5.1 & .9 & -6.2 \\
\hline 37 & 4 & 5.1 & -3.1 & -2.2 \\
\hline 38 & 3 & 5.1 & -.1 & -4.2 \\
\hline 39 & 5 & 5.1 & -.1 & .2 \\
\hline 40 & 4 & 5.1 & -.1 & -2.2 \\
\hline & & & -.1 & \\
\hline & & & -.1 & \\
\hline $\begin{array}{c}\Sigma \mathrm{n}_{2} \\
= \\
40\end{array}$ & $\begin{array}{c}\Sigma \mathrm{n}_{1}= \\
174\end{array}$ & $\Sigma \mathrm{n}_{1}=5,1$ & & $\Sigma \mathrm{n}_{1}=-16.83$ \\
\hline
\end{tabular}

$$
\begin{aligned}
\mathrm{x} & =\frac{\sum \mathrm{x}}{\mathrm{n}} \\
& =\frac{204}{40} \\
\mathrm{~S}_{1}{ }^{2} & =\frac{\sum\left(\mathrm{x}_{1}-\mathrm{x}_{2}\right)^{2}}{\mathrm{n}-1} \\
& =\frac{(204-132.65)}{40-1} \\
& =\frac{107.35}{39} \\
& =2.752 \\
& =\sqrt{2.752} \\
& =1.659
\end{aligned}
$$

CELTIC: A Journal of Culture, English a Teaching, Literature \& Linguistics

ISSN: 2356-0401, E-ISSN: 2621-9158, VOL. 6, NO. 1, June 2019. 
Table 5. Calculation of the Result of the Post-test in Control Group $\left(\mathrm{x}_{2}\right)$

\begin{tabular}{|c|c|c|r|r|}
\hline $\mathrm{n}$ & $\mathrm{X}_{2}$ & $\overline{\mathrm{x}}_{2}$ & $\mathrm{x}_{2}-\overline{\mathrm{x}}_{2}$ & $\left(\mathrm{x}_{2}-\overline{\mathrm{x}}_{2}\right)^{2}$ \\
\hline \hline 1 & 5 & 4.125 & -1.125 & -2.25 \\
2 & 4 & 4.125 & -.125 & -.025 \\
3 & 5 & 4.125 & .875 & .765 \\
4 & 3 & 4.125 & -.125 & -2.25 \\
5 & 5 & 4.125 & .875 & .765 \\
6 & 2 & 4.125 & -2.215 & -4.25 \\
7 & 4 & 4.125 & -.125 & -.025 \\
8 & 5 & 4.125 & .875 & .765 \\
9 & 4 & 4.125 & -.125 & -.25 \\
10 & 5 & 4.125 & .875 & .765 \\
11 & 0 & 4.125 & 0 & 0 \\
12 & 4 & 4.125 & -.125 & -.25 \\
13 & 5 & 4.125 & .875 & .765 \\
14 & 4 & 4.125 & -.125 & -.25 \\
15 & 5 & 4.125 & .875 & .765 \\
16 & 4 & 4.125 & -.125 & -.25 \\
17 & 4 & 4.125 & -.125 & -.025 \\
18 & 4 & 4.125 & -.125 & -.025 \\
19 & 2 & 4.125 & -2.125 & -4.25 \\
20 & 3 & 4.125 & -1.125 & -2.25 \\
21 & 2 & 4.125 & -2.125 & -4.25 \\
22 & 3 & 4.125 & -1.125 & -2.25 \\
23 & 3 & 4.125 & -1.125 & -2.25 \\
24 & 4 & 4.125 & -.125 & -.25 \\
25 & 5 & 4.125 & .875 & .765 \\
26 & 4 & 4.125 & -.125 & -.25 \\
27 & 3 & 4.125 & -1.125 & -.25 \\
28 & 3 & 4.125 & -1.125 & -2.25 \\
29 & 5 & 4.125 & .875 & 62.25 \\
30 & 5 & 4.125 & .875 & .765 \\
\hline
\end{tabular}

$$
\begin{aligned}
\mathrm{x} & =\frac{\sum \mathrm{x}}{\mathrm{n}} \\
& =\frac{165}{40} \\
\mathrm{~S}_{1}{ }^{2} & =\frac{\sum\left(\mathrm{x}_{2}-\mathrm{x}_{2}\right)^{2}}{\mathrm{n}-1} \\
& =\frac{(165-17.015)}{40-1} \\
& =\frac{147.98}{39} \\
& =2.794
\end{aligned}
$$$$
\mathrm{S}_{1}^{2}=\sqrt{3.794}
$$$$
=1.947
$$

Table 5 continued

\begin{tabular}{|c|c|c|r|r|}
\hline $\mathrm{n}$ & $\mathrm{X}_{2}$ & $\overline{\mathrm{x}}_{2}$ & $\mathrm{x}_{2}-\overline{\mathrm{x}}_{2}$ & $\left.\mathrm{x}_{2}-\overline{\mathrm{x}}_{2}\right)^{2}$ \\
\hline \hline 31 & 3 & 4.125 & -.125 & -2.25 \\
32 & 6 & 4.125 & .875 & 3.51 \\
33 & 6 & 4.125 & 1.875 & 3.51 \\
34 & 6 & 4.125 & 1.875 & 3.51 \\
35 & 6 & 4.125 & 1.875 & -4.25 \\
36 & 2 & 4.125 & -2.125 & 3.51 \\
37 & 6 & 4.125 & 1.875 & .765 \\
38 & 5 & 4.125 & .875 & 3.51 \\
39 & 6 & 4.125 & 1.875 & .765 \\
40 & 5 & 4.125 & .875 & -5.275 \\
\hline$\Sigma \mathrm{n}_{2}$ & $\Sigma \mathrm{x}_{2}$ & $\sum \mathrm{x}_{2}=4.125$ & & $\sum\left(\mathrm{x}_{2}-\overline{\mathrm{x}}_{2}\right)^{2}=5.275$ \\
\hline
\end{tabular}

CELTIC: A Journal of Culture, English a Teaching, Literature \& Linguistics

ISSN: 2356-0401, E-ISSN: 2621-9158, VOL. 6, NO. 1, June 2019. 


\begin{tabular}{|c|c|l|l|l|}
\hline$=$ & $=$ & & & \\
40 & 165 & & & \\
\hline
\end{tabular}

In this study, the Experimental group was expected to score higher than the Control group. Also, to test whether there was a significant difference between these two groups, $t$ can be calculated by inserting the values from table 4 and table 5 into the following formula:

$$
\begin{aligned}
& \left.\mathrm{t}_{\mathrm{x}-\mathrm{x}} \text { (obs. }\right)=\frac{\mathrm{x}_{1}-\mathrm{x}_{2}}{\sqrt{\left[\frac{\left(\mathrm{n}_{1}-1\right) \mathrm{S}_{1}^{2}+\left(\mathrm{n}_{2}-1\right) \mathrm{S}_{2}^{2}}{\mathrm{n}_{1}+\mathrm{n}_{2}-2}\right]\left[\frac{1}{\mathrm{n}_{1}}+\frac{1}{\mathrm{n}_{2}}\right]}} \\
& =\frac{5.1-4.125}{\sqrt{\left[\frac{(40-1) 2.752+(40-1) 3.794}{40+40-2}\right]\left[\frac{1}{40}+\frac{1}{40}\right]}} \\
& =\frac{5.1-4.125}{\sqrt{\left[\frac{(39) 2.752+(39) 3.794}{78}\right]\left[\frac{2}{40}\right]}} \\
& =\frac{5.1-4.125}{\sqrt{\left[\frac{107.328+147.966}{78}\right]\left[\frac{2}{40}\right]}} \\
& \mathrm{t}_{\mathrm{x}_{1}-\mathrm{x}_{2}}(\text { obs. })=\frac{.975}{\sqrt{\left[\frac{255.294}{78}\right]\left[\frac{2}{40}\right]}} \\
& =\frac{.975}{\sqrt{[3.275][.05]}} \\
& =\frac{.975}{\sqrt{.163}}=\frac{.975}{.404} \\
& =2.413
\end{aligned}
$$

In order to decide whether or not reject the null hypothesis, the observed value of $t$ $(2,000)$ was compared to the critical value of $t$. Since there is a different critical value of $t$ for each degree of freedom, the first step is to determine the number of degree of freedom $(d f)$. The degree of freedom for the critical value of $t$ is:

$$
\begin{aligned}
d f & =\mathrm{n}-2 \\
& =80-2 \\
& =78
\end{aligned}
$$


In table C (See appendix), it is found that the critical value of $\mathrm{t}$ for $78 \mathrm{df}$ at the level of significance .05 is 2,000 . This means that since $t_{\text {obs }}$ is higher than the $t_{\text {crit }}(2,413>$ 2,000), the null hypothesis is rejected. Therefore, the alternative hypothesis is not rejected.

\section{CONCLUSIONS AND SUGGESTION \\ Conclusions}

Based on the discussion, the following points have been concluded:

The scores of the students in the Experimental group were in majority higher than those in the Control group. This means that there was a significant difference between the number of words which were memorized by the students in top position (primacy position) and that in the last position (recency position). Words that were memorized by the students from the word list in the top position were easier to recall than those in the last position. The students could better remember the words in the top position than those in the last position; in other words, primacy effect was more dominant than recency effect.

\section{Suggestions}

As the completion of this research, the following suggestions have been pointed out: Theoreticians can use this research to support their theory which is stated "words in the top position are easy to recall than words in the last position". In applying memorizing words to teach the vocabulary of a foreign language. Teachers especially English teachers have to consider the positions of words. This means that difficult words are put in the top position, so that students can recall them better.

\section{REFERENCES}

Good, T.L \& Brophy, J.E. (1982). Educational Psychology. New York: Prentice Hall

Holtzman, W.H. (1978). Introduction to Psychology. New York: Harper \& Row, Publishers.

Mayer, R. E. (1998). The Cambridge handout of multimedia learning. New York: Cambridge University Press.

Ruch, J.C. (1984). Psychology: The Personal Science. Belmont: Wadsworth Publishing Company.

Shavelson, R. (1981). Statistical Reasoning for the Behavioral Science. Boston: Allyn \& Bacon.

Ur, P. (1996). A Course in Language Teaching: Practice and Theory. New York: Cambridge University Press.

Walker, J.T (1996). The Psychology of Learning. Upper Saddle River: Prentice Hall Inc. 